\title{
micromachines
}

ISSN 2072-666X

www.mdpi.com/journal/micromachines

Review

\section{Approaches and Challenges of Engineering Implantable Microelectromechanical Systems (MEMS) Drug Delivery Systems for in Vitro and in Vivo Applications}

\section{Danny Jian Hang Tng ${ }^{1}$, Rui Hu ${ }^{1}$, Peiyi Song ${ }^{1}$, Indrajit Roy ${ }^{2}$ and Ken-Tye Yong ${ }^{1, *}$}

1 School of Electrical and Electronic Engineering, Nanyang Technological University, Singapore 639798, Singapore

2 Department of Chemistry, University of Delhi, Delhi 110 007, India

* Author to whom correspondence should be addressed; E-Mail: ktyong@ntu.edu.sg; Tel.: +65-6790-5444.

Received: 13 September 2012; in revised form: 2 November 2012 / Accepted: 7 November 2012 / Published: 14 November 2012

\begin{abstract}
Despite the advancements made in drug delivery systems over the years, many challenges remain in drug delivery systems for treating chronic diseases at the personalized medicine level. The current urgent need is to develop novel strategies for targeted therapy of chronic diseases. Due to their unique properties, microelectromechanical systems (MEMS) technology has been recently engineered as implantable drug delivery systems for disease therapy. This review examines the challenges faced in implementing implantable MEMS drug delivery systems in vivo and the solutions available to overcome these challenges.
\end{abstract}

Keywords: MEMS device; drug delivery; biomedical applications; implantable

\section{Introduction}

Microelectromechanical systems (MEMS) is the study of the fabrication of devices in the micro scale utilizing microfabrication techniques as used in integrated circuit fabrication. MEMS devices consist of electrical parts and also moving parts that allow a physical function to be executed in addition to their electrical functions [1]. In the past few years, improvements in MEMS technology has made device miniaturization possible [2] and this technology has started the field of microfluidic systems, which engineer devices in the micrometer scale that can sense, pump, mix and monitor fluids 
at volumes as low as microliters [3]. Many MEMS devices have been made for biological applications and these devices are known as BioMEMS. BioMEMS devices are sensitive and responsive, allowing accurate control to sense and deliver biomolecules in vitro and in vivo. BioMEMS encompasses a large variety of electronic devices such as biosensors, stents, immunoisolation devices, drug delivery systems and injectable devices [1].

Drug delivery systems play an important role in for many healthcare applications. However, conventional drug treatments such as oral administration or injection, have many limitations, such as being unsuitable for long-term treatment, having a narrow drug therapeutic window, utilizing a complex dosing schedule with combination therapy or labile active ingredients, etc. [4]. In recent years, there has been great attention focusing on the usage of microdevices and nanodevices in disease treatment. These devices, such as wirelessly controlled micro-robots which are small enough to be magnetically steered, can penetrate deep within the body and thus potentially function as drug vectors [5-7]. Although these devices present great potential, they do not yet address all the limitations faced by conventional drug treatments. In retrospect, implantable BioMEMS devices which have larger size have shown many advantages in comparison to classic drug delivery methods and will be the focus of this review. For example, the size of the BioMEMS devices is in the micrometer scale, allowing one to implant them into the region of interest for localized drug therapy [8]. These strengths and advantages allow implantable BioMEMS to build onto the foundation laid by conventional drug delivery methods. In particular, drugs delivered with conventional methods such as injection can be used in these implantable BioMEMS devices. These drug formulations can take the form of nanoparticle formulations for cancer therapy [9] to intravenous formulations $[10,11]$ and even other potent substances such as hormones or painkillers [12]. Moreover, sensors can be incorporated into these BioMEMS devices where they can monitor the drug release profile and provide useful information for bioengineers and clinicians to optimize the drug therapy for the patients [8].

In this article, we review and discuss the current state of the art implantable BioMEMS for drug delivery applications. More specifically, we focus our discussion on the several major factors that impact the performance of implantable BioMEMS devices, namely, (i) drug reservoir size and loading volume [13,14], (ii) effective delivery method, (iii) operating time [15,16], (iv) controllability of the device [1] and (v) biocompatibility of the device [17]. Depending on the specific biological applications, the device must be carefully tailored for the personalized medicine used. For example, an appropriate drug volume must be incorporated into the device for sustainable long term release of drug molecules to the disease area.

\section{Drug Reservoir Size and Loading Volume}

One of the unique features of implantable BioMEMS is the tunability of the drug payload that the device can carry. A typical device on average contains a volume of drug ranging from microliters to a few milliliters $[18,19]$. As Implantable BioMEMS have to conform to the physiological constraints imposed by the implantation region, it is impractical to continuously increase the size of the device to accommodate a larger drug reservoir. Staples et al. [20] have reported that the overall size of the device is a major consideration. The device should be as small as possible so as not to cause any negative effects after implantation due to tissues rubbing onto the implant [21]. Furthermore, a small 
device would allow an ease of implantation, requiring only minor surgery and local anesthesia. The converse is also true, where the shape and material of the device would affect its ease of removal. As an example, in ocular implants, the preferred implant device should be relatively thin as mentioned by Lo et al. [13], the thickness of the device must not exceed $2 \mathrm{~mm}$ and it is preferable that the cannula does not exceed $1 \mathrm{~mm}$ in diameter. In other more restrictive areas, such as the neural prostheses in the spine, the device should be 100 times smaller than a pacemaker [22]. This restriction in size places a limitation on the overall loading volume of the device. Therefore, it is important to minimize the overall size of the device and yet achieve a relatively large loading volume for specific therapy applications. There are several approaches which have been used to overcome the low drug payload volume: (i) employ an external reservoir, (ii) use transdermal delivery, (iii) use a refillable reservoir, and (iv) reduce the size of other device parts, such as the power sources and actuating mechanisms, thereby allowing more space for increasing the loading volume of the device.

By separating the reservoir from the drug delivery device, it is possible to reduce the size of the device at the implant site as well as increase the overall loading volume. As a result the device is split into two parts: (i) the implant at the target site and (ii) an external reservoir which is located apart from the target site implant. Both parts are connected via a cannula/catheter (Figure 1). The implant at the target site mainly functions to deliver the drug dose from the external reservoir to the target site and may contain other functions such as sensing and flow control. The external reservoir contains a pump or a type of actuation mechanism which enables it to deliver the drug to the implant at the target site via a cannula/catheter connected between the 2 parts. This approach has been used to treat diabetes mellitus over the past few years as described by Hanaire et al. [23], Lenhard et al. [24] and Staples et al. [4], where the reservoir of an insulin pump is located in the subcutaneous region of the patient rather than implanting it at the target site, allowing it to have more drug available for treatment. However, the disadvantage of this method is that there is a risk of infection at the catheter site as well as the possibility of contact dermatitis of the skin around the drug reservoir [24].

Figure 1. Illustration of a drug delivery device with a separated reservoir embedded under dermal tissue. The drug was pumped through the catheter to the target site where a microvalve was used to regulate the flow rate of the drug.

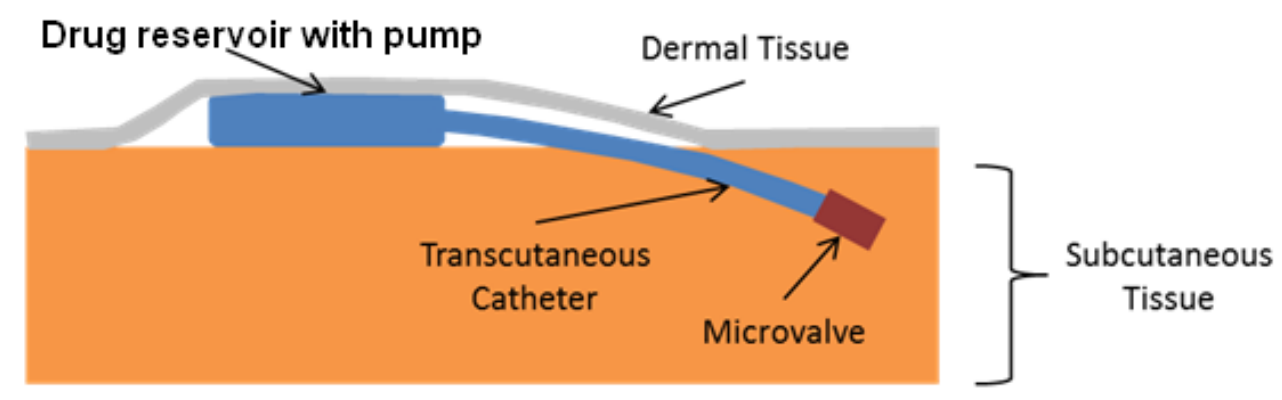

Transdermal drug delivery approach also demonstrates potential to overcome the limitations of a small drug reservoir supply due to the small implant size. This method involves delivering drugs through the skin, is minimally invasive and requires no surgery. Transdermal delivery utilizes MEMS microfabrication technology to create microneedles that help to increase the efficiency of transdermal delivery of drugs [25]. As suggested by Praunitz et al. [26], mirconeedles will increase the skin 
permeability to a wide range of molecules and nanoparticle formulations. There are a broad range of microneedles which include silicon microprobes, glass microcapillaries, and silicon/polysilicon microhypodermic needles that can be used for cellular, local and systemic delivery [27]. These microneedles are integrated into either reservoir type or matrix type patches, which can then be applied onto the skin for transdermal drug delivery. Additionally, they can be used in combination with several active transport methods such as iontophoresis, electro-osmosis, or electroporation for improving the efficiency of drug delivery. Delivery of drugs through the transdermal pathway reduces the dependency for a large reservoir size, as a surface mounted device can be easily replaced or refilled. The limitation however, is that a large bulk of drugs are still not transportable with this method and penetration with microneedles remains relatively shallow within the skin [28].

Another approach which has been successfully demonstrated by Evans et al. [29] and Li et al. [30] is the use of a refillable drug reservoir, thus reducing the need to have an extremely large drug reservoir. The disadvantage is that the refill procedure imposes some risks to the patients, especially if the BioMEMS device is implanted to a fragile and sensitive part of the body such as in the eyes. For example, Li et al. [30] have reported that the refill process requires a needle to be directly inserted into the device which is located at the eye surface, where there is a running risk of accidentally puncturing the surrounding eye tissues. Another issue is that there is no indicator within the BioMEMS reservoirs to inform one that the reservoir is fully filled with drug formulation and the overfilling of the reservoir may result in damaging the device.

Miniaturization of the power source and actuating systems in the device will allow room for a larger drug reservoir without increasing the overall volume of the device. Recent advancements in power supplies for BioMEMS systems demonstrate that it is possible to minimize the power supply components. Conventional methods of supplying electrical power utilize internal Li-ion microbatteries [31]. However, these microbatteries usually occupy a large space in the device, leaving limited space for integrating the other parts into the device. In the past decade, thin film Li-ion batteries with a long cycle life and high charge/discharge capability for their size have been developed [32,33]. These thin film batteries are an excellent candidate to replace microbatteries for powering BioMEMS devices as they take up less space in the device, allowing for a larger drug reservoir to be incorporated. Another approach is to obtain electrical power from external sources. For example, transmitting the power wirelessly to the device via radio frequency, since the wireless radio/magnetic telemetry circuits are smaller in size in comparison to the microbatteries, space is available to be designated to the drug reservoir.

\section{Effective Delivery Methods}

A delivery method is the core technology of the BioMEMS drug delivery device, as it provides the driving force to dispense the stored drug from the reservoir toward the targeted site. In most devices, the delivery method is in the form of an actuator that pressurizes the drug reservoir and releasing the drug formulation through a delivery port. This actuation mechanism can be mechanical in nature, for example, of electrostatic, piezoelectric, thermopneumatic or bimetallic actuation. Alternatively, it can also be non-mechanical, for instance, electroosmotive, electrowetting or evaporation methods [29]. However, non-mechanical methods are highly dependent on the properties of the fluid to be 
transported and therefore it is a challenge for implementing in the body. As a result, mechanical methods are preferred as they provide a general form of fluid manipulation [34]. The actuation method controls the flow rate of the drug formulation when they are pumped out from the device. In order to maximize the drug reservoir volume of the device, the actuation mechanism has to be minimalistic yet effective. Examples of such minimalistic methods include electrochemical method [30], electrothermal method [35] (Figure 2), and passive method [13]. In general, delivery methods for BioMEMS devices fall into three main categories: (i) actuation-less methods, (ii) single chamber actuation methods and (iii) multi-chamber actuation methods.

Actuation-less methods utilize passive mechanisms such as diffusion to perform drug delivery. Typically, these methods rely on a controlled microvalve, causing it to open and close the drug reservoir. The microvalve is usually controlled by an external stimulus, such as by radio frequency or magnetic wave telemetry. A popular microvalve used for this BioMEMS design is a thermosensitive material known as hydrogel. Rahimi et al. [35] have demonstrated that by using radio frequency telemetry, heat can be generated within a coil in contact with the hydrogel microvalves, causing the hydrogel to contract, thus opening the valves for delivering drugs via passive diffusion (Figure 2). The overall size dimension of the device is approximately $5 \mathrm{~mm} \times 5 \mathrm{~mm} \times 4 \mathrm{~mm}$ and has a drug reservoir size of about $78 \mathrm{uL}$. A novel concept of an actuation-less method is proposed by Lo et al. [13] that involves a manually actuated BioMEMS device where one will apply pressure onto the device, thus pressurizing the drug reservoir and releasing the drug solution.

Figure 2. Illustration of a thermoelectric drug delivery device based on thermal sensitive hydrogel, redrawn from reference [35]. (a) Schematic view of the device. The thermal sensitive hydrogel valve lies on top of the heating coil and obstructs the drug release port, sealing the drug reservoir. (b) When the heating coil is switched on, the generated heat causes the hydrogel valve to contract, opening the release port, thus dispensing the drug.

(a)

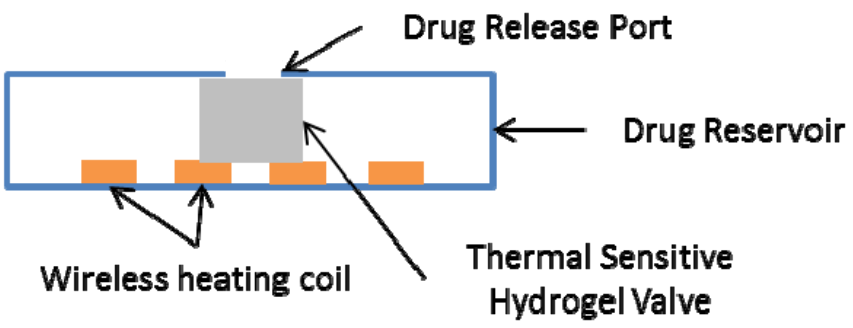

(b)

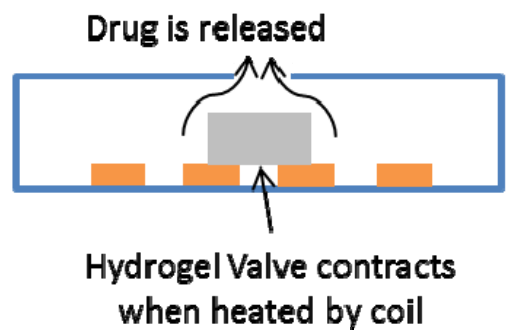

In a single chamber actuation method, the actuation system is housed within the same chamber as the drug reservoir. Since the actuator is built within the drug reservoir, the whole device is more compact. Electrochemical actuators are prime examples of single chamber actuation methods. Li et al. [30] have designed an electrochemical micropump where gas is generated from the electrolysis of water within the drug reservoir (Figure 3). The generated gas then gradually builds up the pressure within the reservoir, pushing the drug solution out through the cannula into the target site. The high pressure created by the gas produces a high flow rate. However, the disadvantage accompanied with this approach is that the electrolysis reaction may induce degradation of the drug within the reservoir [36]. Another single chamber actuation method, utilizes the electrochemical/electrothermal effect in combination with drug microwells. One example is the device proposed by Chung et al. [37], 
which consists of drug formulation containing microwells sealed with thin film gold electrodes. The electrolysis reaction of water within each microwell will not only cause gas bubbles to form which builds up the pressure in the microwell but also has an added effect of dissolving the gold electrode seal. After some time, the dissolved gold electrode will be too thin to resist the pressure buildup, causing it to rupture and releasing the microwell contents rapidly.

Figure 3. Electrochemical implantable BioMEMS pump proposed by Li et al. Electrodes placed at the bottom of the drug reservoir perform the water hydrolysis reaction when there is a potential difference between the positive and negative electrodes. Gas produced from the hydrolysis reaction causes a pressure build up and dispenses the drug. Reprinted with permission from reference [30]. Copyright (C) 2008, Elsevier.

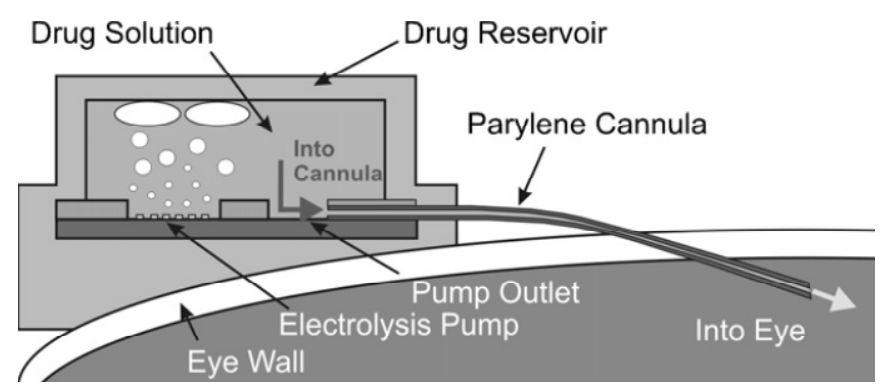

Figure 4. A multi-chamber electrochemical device, adapted for in vivo Applications. (a) Schematic view of the parts of the device. (b) Electrochemical actuation of the bellows. (c) Side view of the device showing its thickness. (d) Photo of prototype model showing the electrode housed within the pump chamber and the drug reservoir lying on top, connected to the cannula. (e) SEM Picture of electrode showing the electrode fingers of thickness $50 \mu \mathrm{m}$ and $50 \mu \mathrm{m}$ spacing.

(a)

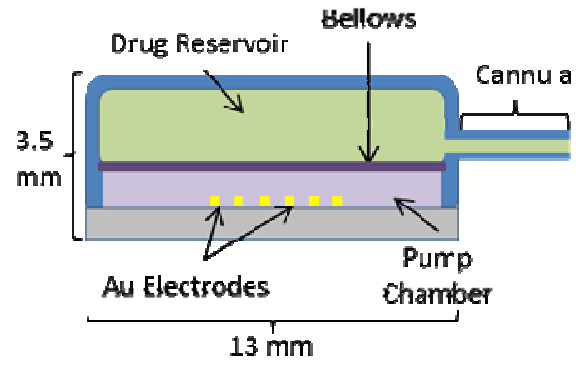

(b)

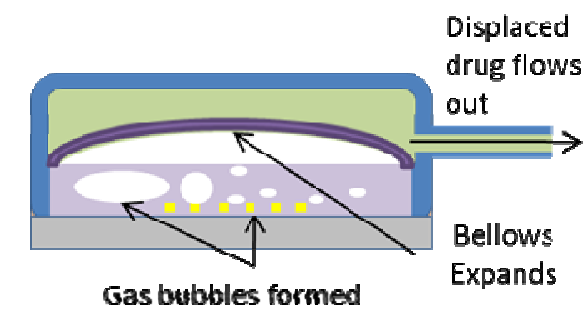

(c)

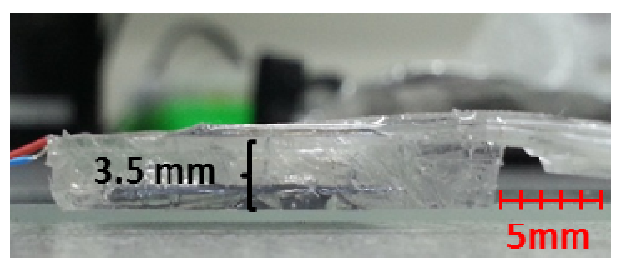

(d)

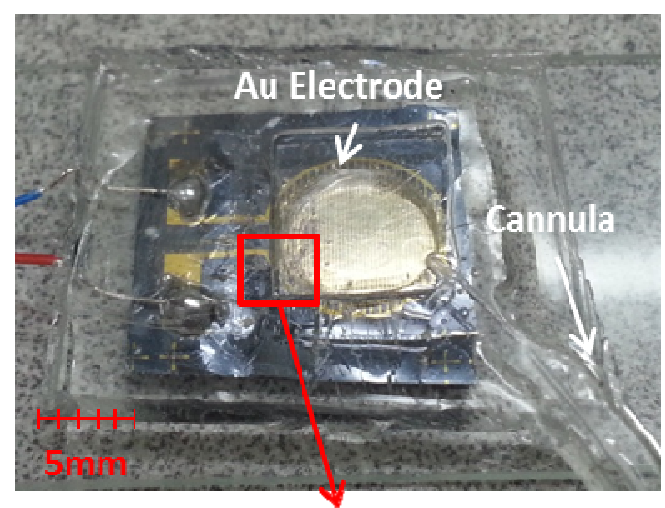

(e)

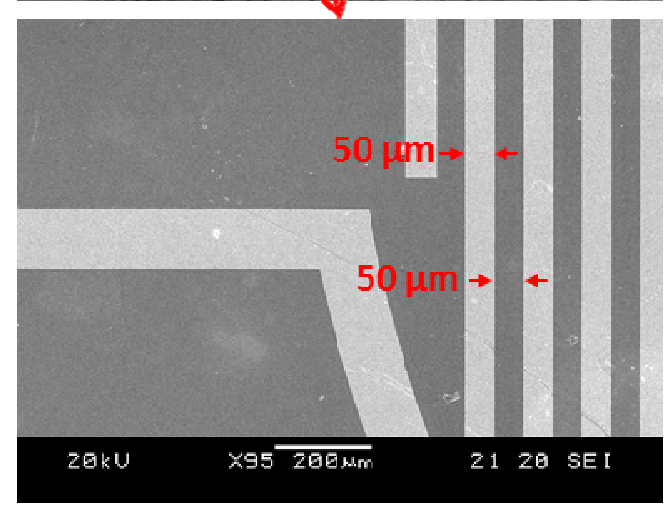


The multi-chamber actuation system is the most complex compared to the other systems. In general, it consists of two or more chambers, where the drug reservoir is separated from the actuator, isolating the two components and preventing cross contamination. The disadvantage of this system is that the drug reservoir volume is significantly reduced due to the number of chambers. Li et al. [36] have successfully implemented a two-chamber system where the electrochemical actuator is housed within an expandable parylene bellows within the drug reservoir. An electrolysis reaction takes place within the bellows converting the water contained into gas, expanding the bellows and thus displacing the drug out of the reservoir. We present a similar approach (Figure 4), a multi-chamber, refillable device made of polydimethylsiloxane (PDMS), adapted for in vivo applications. The device contains an electrochemically actuated bellows, has a slim profile (3.5 $\mathrm{mm}$ thick) for ease of implantation and relatively high flow rate $(0.4 \mu \mathrm{L} / \mathrm{min}$ at $3 \mathrm{~V})$ due to its modified cannula position at the corner of the reservoir, reducing the head loss of the fluid as it flows. The device is currently being tested with suitable drugs for its effectiveness in treating cancer cells in vitro.

\section{Long Operation Time}

Equally as important as the loading volume, implantable BioMEMS drug delivery systems must also be power efficient for their intended long term operation and drug dosing [38]. One of the greatest advantages of BioMEMS in the treatment of chronic diseases is that, in addition to localized/targeted delivery, they are able to provide personalized drug delivery in the form of a continuous pulsatile drug release profile $[2,39,40]$. This personalized drug release profile is specifically designed to maximize the therapeutic effects of the drug while limiting the side effects associated with overdose in conventional drug therapies [40]. In order to perform this mode of treatment with patients, implantable BioMEMS must be able to remain operable for a long period of time such that long term personalized medication can be used to treat these chronic diseases. Inherently, due to the small size of these implantable BioMEMS, powering the device for a long time is an issue [41]. However, several methods have been investigated to overcome this issue: (i) the adoption of low power actuation mechanisms [33] and/or (ii) increasing the battery life of the device by enabling the internal batteries to be recharged [42-44] or using wireless power [35,45].

In order to manage chronic diseases, implantable BioMEMS have to adopt a power efficient design such as the drug delivery pump proposed by Chung et al. [37] where only 8-12 V is required, compared to other high power devices that require $80-120 \mathrm{~V}$ as reported by Geipei et al. [46]. The recent trend is in the development of low-power active components such as valves and actuators [47]. As discussed by Dissanayake et al. [48], there are some low power actuators available that can be used as battery-less actuators, relying on telemetry to function.

Another method to increase the operation time of the device is to increase its battery life. Recently, several novel approaches have been used to power BioMEMS devices for biological applications. These approaches involve the use of thin film batteries as discussed by Nathan et al. [31], wireless power transmission as proposed by Smith et al. [49] and battery recharging through the drug refill port as reported by Evans et al. [29]. Wireless power transmission has been demonstrated successfully and has a strong potential for powering implantable BioMEMS to increase operation time. Simons et al. [15] have proposed a miniature inductor and a pick up antenna in order to wirelessly 
power a biosensor over short distances. Following this similar approach, Rao et al. [32] have developed a method for powering a micropump for their BioMEMS device. With the use of radio frequency telemetry to drive an in-plane silicon pump, the micropump was able to achieve an average flow rate of $85 \mu \mathrm{L} / \mathrm{min}$, which is comparable to the pumps using microbatteries. Another example of an actuator being driven wirelessly is the electrochemical actuator designed by Li et al. [36], where a radio frequency signal is used to set up a current and voltage across electrodes for the water hydrolysis reaction to occur and produce gas. The gas performs the actuation action and pumps the drug out from the reservoir with a flow rate of $10 \mu \mathrm{L} / \mathrm{min}$. Other than radio frequency telemetry, other forms of wireless energy transfer methods have been adopted, such as using magnetic fields to transmit wireless power via magnetic telemetry as demonstrated by Najafi et al. [50]. These wirelessly powered devices have significantly increased the operating time of the device. However, since the communication range is limited, devices which are implanted deep within the body may not be able to receive the telemetric signal due to the attenuation of the signal imposed by the tissue. Studies by Vaillancourt et al. [51] have demonstrated that telemetric signals such as radio frequency waves experience electric field strength attenuation by up to $15 \mathrm{~dB}$ as they are passing through the biological tissue. This suggests that wirelessly powered devices have to account for this attenuation loss if a device is implanted deep into the body. Besides wireless power transmission, it is also possible to recharge internal microbatteries through their refill port as demonstrated by Evans et al. [50]. However, one of the major complications with using this method is that the recharge needle must be kept inserted throughout the duration of recharging, where the movement of the patient might disrupt the charging.

\section{Controllability}

The kinetics of drug release within the BioMEMS device can be controlled by electrical signals. In general, there are three ways to control the BioMEMS device, namely, active, passive, and by environmental biological stimulus. Also influencing the controllability of a device would be its ability to evaluate the effectiveness of the delivered drug, that is, the responsiveness of the device. Responsiveness allows greater controllability as it enables the regulation of the active and passive control of the drug delivery device according to the current state of the disease [8].

Active control is where an external stimulus, such as an electrical signal, radio frequency wave or magnetic wave alters the behavior of the drug delivery device and causes it to dispense the drug. The ability to provide active control is one of the unique features of BioMEMS devices for drug delivery applications and much effort has been focused on integrating different programmable elements into the devices [4]. Wireless active control has been engineered mainly by using radio frequency wave telemetry. Prescott et al. [52] have demonstrated that telemetry can be precisely controlled to regulate the release of a drug formulation from a multiwell device. Magnetic field telemetry has also been used to perform wireless control of the implanted devices. Pirmoradi et al. [12] have reported the development of a device whose drug reservoir is sealed with a magnetic membrane that possesses an aperture. Upon applying the magnetic field to the device, this will cause deformation to the membrane and consequently leading to the release of the drug molecules. The device is actuated by the magnetic field and is capable of releasing a controlled amount for up to 35 days. Another type of active wireless control at the material/molecular level has been previously discussed by Timko et al. [53]. Special 
materials such as gold nanostructures, poly NIPAm composities, liposomes and polymers can be bonded with drug molecules. These materials will release the bonded drug molecules under the influence of a specific signal such as visible light, near-infrared light, ultrasound or magnetic fields.

The second type of control is known as passive control, where the device is pre-programmed to release a certain drug dosage profile. This pre-programming can be achieved either by using active transport mechanisms or by other passive mechanisms such as diffusion. However, although these systems are generally less complex in terms of fabrication and design, they have less precision for controlling the drug delivery [4]. Grayson et al. [54] have demonstrated that a degradable polymer chip containing many membrane covered reservoirs is able to be used for such passive controlled drug delivery applications. The device releases the drug at specific time points based on the property of the reservoir membrane (e.g., degradation rate of the membrane). The device is thus considered to be pre-programed, where the drug release profile can be controlled by tailoring the degradation rate of the membrane.

The third approach is the use of environmental biological stimulus to control the device. Desai et al. [55] have shown that it is possible to use MEMS microfabrication technology to create silicon diffusion membranes with well-controlled uniform pore sizes for use in constructing biocapsules. Cells encapsulated within the biocapsules dictate the production of the desired substance based on the biological stimulants passing through the silicon membranes to the cells. The produced substance subsequently diffuses through the same membrane and is released into the body.

Once the drug is released into the body, the responsiveness of non-preprogrammed BioMEMS drug delivery systems will take over the subsequent doses. A responsive drug delivery system would allow the drug dosage to be specifically tuned according to the type and state of the disease within the individual, providing personalized drug delivery, maximizing the therapeutic effects of the drugs and reducing the overdose induced side-effects [40]. These systems have biosensors which allow the BioMEMS drug delivery devices to evaluate the efficiency of the administered drug by monitoring specific marker molecules of the disease being treated [56]. Therefore, any inhibition or regression of the treated disease can be measured and the drug delivery device can adjust its subsequent dose by controlling the drug release through passive or active control as discussed above. One area of great interest are flow control systems utilizing responsive hydrogels, as they are biocompatible and can be specifically engineered to respond a variety of factors and marker molecules within the blood such as temperature, $\mathrm{pH}$, saccharide and antigen concentration [57,58]. These factors fluctuate as the disease being treated, and thereby allowing the drug delivery system to evaluate the effectiveness of the drug administered [58-61].

\section{Biocompatibility}

The biocompatibility of the implanted BioMEMS devices is an important factor to be considered carefully since they will be implanted in the body for a long period of time and they will interact with tissues in the body [21]. There are a few factors to be considered when designing an implantable BioMEMS device: (i) the impact of the implantable device on the immune response, (ii) minimizing biofouling on the implanted device, (iii) the physical effect of the implant to the surrounding tissues and (iv) the degree of cell adhesion achieved by the implanted device. 
To avoid adverse physiological effects, such as blood and tissue incompatibility due to these implants as discussed by Park et al. [62], the implanted devices must be packaged with biocompatible materials. However, the complication is that biocompatible materials might not always be compatible with the device requirements. Thus, special considerations have to be made when selecting the material for packaging, structural support or hermetically sealing the device. Lo et al. [13] has recently demonstrated that BioMEMS designs can be incorporated with highly biocompatible silicones and polymers such as polyetheretherketone (PEEK) and polyimide (PL) to serve as a baseplate for providing structural support. In addition, the baseplate prevents the puncturing of the device during the refill process as a needle is used. For device packaging, some common biomaterials are PDMS and poly(tetramethylene oxide) (PTMO). These materials are shown to have high biocompatibility [63,64] and they are widely used in implantable devices [48]. A PDMS packaged BioMEMS drug delivery device has been demonstrated and tested by Li et al. [30]. However, in other situations where a robust housing is required, PDMS would not be an appropriate material as it is too soft, deforms readily under mechanical stress, is prone to rupture and leaking. Harder materials would be needed in these cases, as in the case of pacemakers, which are enclosed in hermetically welded titanium canisters to prevent any moisture from damaging the electronic components [65]. For the injectable/implantable muscle microstimulators, both hermatic sealing and insulation are important for them to work efficiently. These systems are usually packaged in glass, such as the BION Microstimulators as proposed by Kane et al. [66]. Another important factor of these materials is their stability during and after sterilization. As mentioned by Kotzar et al. [21], the manufacture process of these devices will also include a sterilization step, which may alter the properties of these materials. Therefore, careful selection of sterilization methods must also be selected so as not to affect the device performance. Gamma and steam sterilization for example, do not significantly affect these biomaterials, as studied and reported by Ferrara et al. [67].

Biofouling is another serious problem affecting device performance. In general, these devices will be exposed continuously to the various elements within the body. Over time the biomolecules within the body will react with the materials of the device, possibly leading to the passivation and reduced functionality of the device $[68,69]$. For example, the accumulation of proteins in the pores of the material around the drug output port eventually obstructs it [70]. To prevent biofouling, various types of coatings in addition to the device packaging are used. Unfortunately, with more coatings on the device, the size of the device will increase [71]. A detailed study reported by Voskerician et al. [68] showed that implantable BioMEMS packaged with gold, silicon nitride, silicon dioxide and SU-8 were able to reduce biofouling. Similarly, studies by Schmehl et al. [72] also suggest that a coating of silicon carbide can be used to significantly reduce thrombus formation on the surface of the devices, especially if the device is exposed to blood.

Since the implant is in close proximity with other tissues, the most significant physical effect from the device is that it will exert friction on the neighboring tissues. Therefore, for devices that are implanted into tight tissue regions, techniques such as lubrication can be used to overcome the negative physical effects. Coatings on the packaging might be necessary to improve the wear resistance and decrease friction. This is because at the micro scale, frictional forces between moving bodies will be very significant and this affects the operation of some BioMEMS drug delivery devices [73]. Surface friction can be reduced through the creation of micro-scale patterns on the 
surface of the device during fabrication as demonstrated by Singh et al. [73]. Micro-scale patterns can decrease the friction coefficient of surfaces, reducing the negative effects of friction. From an accelerated wear test, Subash et al. [74] have identified two regimes of wear, adhesion-dominated wear which then leads to third-body wear. Adhesion-dominated wear involves asperity blunting, plastic deformation of asperity peaks and the smearing of finely worn debris into a thin-surface film at the points of contact. This progresses into third-body wear, which involves debris agglomeration and causes material to be removed due to friction. The material removal regions resulted in voids which ultimately contributed to device package failure. Yu et al. [75] have shown that the frictional forces on localized areas can be measured using a microtribometer. These regions can then be locally lubricated with the use of liquid lubrication. Surface micro/non-hierarchical structure designs and thin film lubrication are the two main methods used in BioMEMS to reduce adhesive and frictional problems. As demonstrated by Wang et al. [76], silicon surfaces with micro grooves which were further modified by multiply-alklated cyclopentane thin films improved the tribological properties of the surface significantly.

The flexibility of an implant is another crucial factor which can attribute to inflammation within the body. In some areas of the body which are particularly sensitive, such as in the cerebral region, movement of the tissues against rigid implants can cause sustained cellular response, resulting in inflammation [77] and glial scar formation [78] at the implant site. Instead of rigid silicon wafers, flexible implants fabricated onto biocompatible flexible substrates such as polyimide [79] have shown a significant reduction in the negative physiological impacts caused by rigid implants.

Cellular Adhesion is one more consideration that has to be taken account when integrating the BioMEMS device with the adjacent tissues. The packaging can be coated with special materials to encourage cellular adhesion for higher implant compatibility. Cell adhesion dictates how cells organize themselves to form tissues. There is also a strong correlation between cell adhesion and cell to cell signaling within tissues [80]. Various coatings have been used to increase cell adhesion of BioMEMS devices such as using modified SU-8 photoresist as reported by Yuli Wang et al. [81]. S. Bouaidat et al. [82] have also mentioned the use of phosphorous glass (SiPOC) for cell adhesion in BioMEMS.

\section{Conclusion}

MEMS based implantable drug delivery devices offer many significant advantages as opposed to conventional drug delivery methods. These advantages make implantable BioMEMS devices excellent candidates in the treatment of chronic diseases such as cancer. However, despite their numerous advantages, there are many challenges facing the implementation of these devices for the treatment of any specific disease. For each case, these devices must be specially optimized with regard to their drug reservoir size, relative size, operation lifetime, controllability and biocompatibility. An ideal implantable device must have a large enough drug reservoir to minimize refilling and must also have a small size relative to the region of implantation to reduce its interference with surrounding tissues. Long device lifetime is also required so as to reduce the complications associated with servicing or replacing these implants. Having a high degree of controllability enables the doctors to tailor the drug dosage as treatment progresses. Lastly, biocompatibility of the device will ensure that there are no ill 
effects due to the long term implantation of the BioMEMS device within the body. Development is required in these areas especially in terms of small, controlled actuation mechanisms in order to boost the effectiveness of these devices.

\section{Acknowledgement}

This work was supported by the Singapore Ministry of Education (Tier 1 M4010360.040 RG29/10) and Nanyang Technological University (start-up grant no. M4080141.040).

\section{References}

1. Grayson, A.C.R.; Shawgo, R.S.; Johnson, A.M.; Flynn, N.T.; Yawen, L.I.; Cima, M.J.; Langer, R. A BioMEMS review: MEMS technology for physiologically integrated devices. Proc. IEEE 2004, 92, 6-21.

2. Elman, N.M.; Upadhyay, U.M. Medical applications of implantable drug delivery microdevices based on MEMS (Micro-Electro-Mechanical-Systems). Curr. Pharm. Biotechnol. 2010, 11, 398-403.

3. Nisar, A.; Afzulpurkar, N.; Mahaisavariya, B.; Tuantranont, A. MEMS-based micropumps in drug delivery and biomedical applications. Sens. Actuator. B 2008, 130, 917-942.

4. Staples, M.; Daniel, K.; Cima, M.; Langer, R. Application of micro- and nano-electromechanical devices to drug delivery. Pharm. Res. 2006, 23, 847-863.

5. Park, S.; Park, J.O. Frontier Research Program on Biomedical Microrobot for Intravascular Therapy. In 2nd IEEE RAS \& EMBS International Conference on Biomedical Robotics and Biomechatronics, BioRob 2008, Scottsdale, AZ, USA, 19-22 October 2008; pp. 360-365.

6. Nelson, B.J. Towards Nanorobots. In International Conference of Solid-State Sensors, Actuators and Microsystems, 2009, TRANSDUCERS 2009, Denver, CO, USA, 21-25 June 2009; pp. 2155-2159.

7. Shuxiang, G.; Sawamoto, J.; Qingxue, P. A Novel Type of Microrobot for Biomedical Application. In 2005 IEEE/RSJ International Conference on Intelligent Robots and Systems, IROS 2005, Edmonton, Alberta, Canada, 2-6 August 2005; pp. 1047-1052.

8. LaVan, D.A.; McGuire, T.; Langer, R. Small-scale systems for in vivo drug delivery. Nat. Biotech. 2003, 21, 1184-1191.

9. Gensler, H.; Sheybani, R.; Po-Ying, L.; Lo, R.; Zhu, S.; Ken-Tye, Y.; Roy, I.; Prasad, P.N.; Masood, R.; Sinha, U.K.; Meng, E. Implantable MEMS Drug Delivery Device for Cancer Radiation Reduction. In IEEE 23rd International Conference on Micro Electro Mechanical Systems (MEMS), Wanchai, Hong Kong, 24-28 January 2010; pp. 23-26.

10. Li, Y.; Hong Linh Ho, D.; Tyler, B.; Williams, T.; Tupper, M.; Langer, R.; Brem, H.; Cima, M.J. In vivo delivery of BCNU from a MEMS device to a tumor model. J. Control. Release 2005, 106, $138-145$.

11. Li, Y.; Shawgo, R.S.; Tyler, B.; Henderson, P.T.; Vogel, J.S.; Rosenberg, A.; Storm, P.B.; Langer, R.; Brem, H.; Cima, M.J. In vivo release from a drug delivery MEMS device. J. Control. Release 2004, 100, 211-219. 
12. Pirmoradi, F.N.; Jackson, J.; Burt, H.; Chiao, M. Delivery of an Anti-Cancer Drug from a Magnetically Controlled MEMS Device Show Cytotoxicity in PC3 and HUVEC Cells. In the 16th International Conference on Solid-State Sensors, Actuators and Microsystems (TRANSDUCERS 2011), Beijing, China, 5-9 June 2011; pp. 2831-2834.

13. Lo, R.; Li, P.-Y.; Saati, S.; Agrawal, R.; Humayun, M.; Meng, E. A passive MEMS drug delivery pump for treatment of ocular diseases. Biomed. Microdevices 2009, 11, 959-970.

14. Cao, L.; Mantell, S.; Polla, D. Design and simulation of an implantable medical drug delivery system using microelectromechanical systems technology. Sens. Actuator. A 2001, 94, 117-125.

15. Simons, R.N.; Hall, D.G.; Miranda, F.A. RF Telemetry System for an Implantable Bio-MEMS Sensor. In IEEE MTT-S International Microwave Symposium Digest, Forth Worth, TX, USA, 6-11 June 2004; Volume 1433, pp. 1433-1436.

16. Jackson, N.; Muthuswamy, J. Flexible chip-scale package and interconnect for implantable MEMS movable microelectrodes for the brain. J. Microelectromechanical. Syst. 2009, 18, 396-404.

17. Abel, P.U.; von Woedtke, T. Biosensors for in vivo glucose measurement: Can we cross the experimental stage. Biosens. Bioelectron. 2002, 17, 1059-1070.

18. Elman, N.; Ho Duc, H.; Cima, M. An implantable MEMS drug delivery device for rapid delivery in ambulatory emergency care. Biomed. Microdevices 2009, 11, 625-631.

19. Evans, A.; Park, J.; Chiravuri, S.; Gianchandani, Y. A low power, microvalve regulated architecture for drug delivery systems. Biomed. Microdevices 2010, 12, 159-168.

20. Staples, M. Microchips and controlled-release drug reservoirs. WIRs Nanomed. Nanobiotechnol. 2010, 2, 400-417.

21. Kotzar, G.; Freas, M.; Abel, P.; Fleischman, A.; Roy, S.; Zorman, C.; Moran, J.M.; Melzak, J. Evaluation of MEMS materials of construction for implantable medical devices. Biomaterials 2002, 23, 2737-2750.

22. Richards Grayson, A.C.; Scheidt Shawgo, R.; Li, Y.; Cima, M.J. Electronic MEMS for triggered delivery. Advan. Drug Delivery Rev. 2004, 56, 173-184.

23. Hanaire, H.; Lassmann-Vague, V.; Jeandidier, N.; Renard, E.; Tubiana-Rufi, N.; Vambergue, A.; Raccah, D.; Pinget, M.; Guerci, B. Treatment of diabetes mellitus using an external insulin pump: The state of the art. Diabetes Metab. 2008, 34, 401-423.

24. Lenhard, M.J.; Reeves, G.D. Continuous subcutaneous insulin infusion: A comprehensive review of insulin pump therapy. Arch. Intern. Med. 2001, 161, 2293-2300.

25. Park, J.-H.; Allen, M.; Prausnitz, M. Polymer microneedles for controlled-release drug delivery. Pharm. Res. 2006, 23, 1008-1019.

26. Mark R,P. Microneedles for transdermal drug delivery. Advan. Drug Delivery Rev. 2004, 56, 581-587.

27. McAllister, D.V.; Allen, M.G.; Prausnitz, M.R. Microfabricated microneedles for gene and drug delivery. Annu. Rev. Biomed. Eng. 2000, 2, 289-313.

28. Prausnitz, M.R.; Mitragotri, S.; Langer, R. Current status and future potential of transdermal drug delivery. Nat. Rev. Drug Discov. 2004, 3, 115-124.

29. Evans, A.; Chiravuri, S.; Gianchandani, Y. Transdermal power transfer for recharging implanted drug delivery devices via the refill port. Biomed. Microdevices 2010, 12, 179-185. 
30. Li, P.-Y.; Shih, J.; Lo, R.; Saati, S.; Agrawal, R.; Humayun, M.S.; Tai, Y.-C.; Meng, E. An electrochemical intraocular drug delivery device. Sens. Actuator. A 2008, 143, 41-48.

31. Nathan, M. Microbattery technologies for miniaturized implantable medical devices. Curr. Pharm. Biotechnol. 2010, 11, 404-410.

32. Smitha M.N.; Rao, A.M.; Popa, D.O.; Chiao, J.C.; Ativanichayaphong, T.; Sin, J.; Stephanou. H.E. MEMS-based implantable drug delivery system. In VII International Conference on Micro electro Mechanical Systems, 2005 TexMEMS, E1 Paso, TX, USA, 21-22 September 2005.

33. Inke, J.; Lucas, R.; Leonard, H.; Hedley, H.; Vijay, V.; Chris, B.; Simon, M.; Stefan, E.; David, S.; Said, A.-S.; Derek, A. Wireless RF communication in biomedical applications. Smart Mater. Struct. 2008, 17, No. 015050.

34. Studer, V.; Hang, G.; Pandolfi, A.; Ortiz, M.; Anderson, W.F.; Quake, S.R. Scaling properties of a low-actuation pressure microfluidic valve. J. Appl. Phys. 2004, 95, 393-398.

35. Rahimi, S.; Sarraf, E.; Wong, G.; Takahata, K. Implantable drug delivery device using frequencycontrolled wireless hydrogel microvalves. Biomed. Microdevices 2011, 13, 267-277.

36. Po-Ying, L.; Sheybani, R.; Gutierrez, C.A.; Kuo, J.T.W.; Meng, E. A parylene bellows electrochemical actuator. J. Microelectromechanical. Syst. 2010, 19, 215-228.

37. Chung, A.; Huh, Y.; Erickson, D. A robust, electrochemically driven microwell drug delivery system for controlled vasopressin release. Biomed. Microdevices 2009, 11, 861-867.

38. Shawgo, R.S.; Richards Grayson, A.C.; Li, Y.; Cima, M.J. BioMEMS for drug delivery. Curr. Opin. Solid State Mat. Sci. 2002, 6, 329-334.

39. Tsai, N.C.; Sue, C.Y. Review of MEMS-based drug delivery and dosing systems. Sens. Actuator. A 2007, 134, 555-564.

40. Gensler, H.; Sheybani, R.; Li, P.Y.; Lo Mann, R.; Meng, E. An implantable MEMS micropump system for drug delivery in small animals. Biomed. Microdevices 2012, 14, 483-496.

41. Tsai, N.-C.; Sue, C.-Y. Review of MEMS-based drug delivery and dosing systems. Sens. Actuator. A 2007, 134, 555-564.

42. Humble, P.H.; Harb, J.N.; LaFollette, R. Microscopic nickel-zinc batteries for use in autonomous microsystems. J. Electrochem. Soc. 2001, 148, A1357-A1361.

43. Nathan, M.; Golodnitsky, D.; Yufit, V.; Strauss, E.; Ripenbein, T.; Shechtman, I.; Menkin, S.; Peled, E. Three-dimensional thin-film Li-ion microbatteries for autonomous MEMS. J. Microelectromechanical. Syst. 2005, 14, 879-885.

44. Goto, K.; Nakagawa, T.; Nakamura, O.; Kawata, S. An implantable power supply with an optically rechargeable lithium battery. IEEE Trans. Biomed. Eng. 2001, 48, 830-833.

45. Tang, T.B.; Smith, S.; Flynn, B.W.; Stevenson, J.T.M.; Gundlach, A.M.; Reekie, H.M.; Murray, A.F.; Renshaw, D.; Dhillon, B.; Ohtori, A.; Inoue, Y.; Terry, J.G.; Walton, A.J. Implementation of wireless power transfer and communications for an implantable ocular drug delivery system. IET Nanobiotechnol. 2008, 2, 72-79.

46. Geipel, A.; Goldschmidtboeing, F.; Jantscheff, P.; Esser, N.; Massing, U.; Woias, P. Design of an implantable active microport system for patient specific drug release. Biomed. Microdevices $\mathbf{2 0 0 8}$, 10, 469-478.

47. Don, W.D.; Said, A.-S.; Derek, A. Modelling and simulation of wirelessly and securely interrogated low-powered actuators for bio-MEMS. Smart Mat. Struct. 2011, 20, 015025. 
48. Defrère, S.; Mestagdt, M.; Riva, R.; Krier, F.; van Langendonckt, A.; Drion, P.; Jérôme, C.; Evrard, B.; Dehoux, J.-P.; Foidart, J.-M.; Donnez, J. In vivo biocompatibility of three potential intraperitoneal implants. Macromol. Biosci. 2011, 11, 1336-1345.

49. Smith, S.; Tang, T.B.; Terry, J.G.; Stevenson, J.T.M.; Flynn, B.W.; Reekie, H.M.; Murray, A.F.; Gundlach, A.M.; Renshaw, D.; Dhillon, B.; Ohtori, A.; Inoue, Y.; Walton, A.J. Development of a miniaturised drug delivery system with wireless power transfer and communication. IET Nanobiotechnol. 2007, 1, 80-86.

50. Najafi, N.; Ludomirsky, A. Initial animal studies of a wireless, batteryless, MEMS implant for cardiovascular applications. Biomed. Microdevices 2004, 6, 61-65.

51. Vaillancourt, P.; Djemouai, A.; Harvey, J.F.; Sawan, M. EM Radiation Behavior upon Biological Tissues in a Radio-Frequency Power Transfer Link for a Cortical Visual Implant. In Proceedings of the 19th Annual International Conference of the IEEE on Engineering in Medicine and Biology Society, Chicago, IL, USA, 30 October-2 November 1997; Volume 2496, pp. 2499-2502.

52. Prescott, J.H.; Lipka, S.; Baldwin, S.; Sheppard, N.F.; Maloney, J.M.; Coppeta, J.; Yomtov, B.; Staples, M.A.; Santini, J.T. Chronic, programmed polypeptide delivery from an implanted, multireservoir microchip device. Nat. Biotech. 2006, 24, 437-438.

53. Timko, B.P.; Dvir, T.; Kohane, D.S. Remotely triggerable drug delivery systems. Adv. Mat. 2010, $22,4925-4943$.

54. Grayson, A.C.R.; Choi, I.S.; Tyler, B.M.; Wang, P.P.; Brem, H.; Cima, M.J.; Langer, R. Multi-pulse drug delivery from a resorbable polymeric microchip device. Nat. Mater. 2003, 2, 767-772.

55. Desai, T.A.; Hansford, D.; Ferrari, M. Characterization of micromachined silicon membranes for immunoisolation and bioseparation applications. J. Membrane Sci. 1999, 159, 221-231.

56. Deo, S.K.; Moschou, E.A.; Peteu, S.F.; Bachas, L.G.; Daunert, S.; Eisenhardt, P.E.; Madou, M.J. Peer reviewed: Responsive drug delivery systems. Anal. Chem. 2003, 75, 206A-213A.

57. Qiu, Y.; Park, K. Environment-sensitive hydrogels for drug delivery. Adv. Drug Delivery Rev. 2012, in press, http://dx.doi.org/10.1016/j.addr.2012.09.024.

58. Miyata, T.; Asami, N.; Uragami, T. A reversibly antigen-responsive hydrogel. Nature 1999, 399, 766-769.

59. Eddington, D.T.; Beebe, D.J. Flow control with hydrogels. Adv. Drug Delivery Rev. 2004, 56, 199-210.

60. Chen, J.; Chu, M.; Koulajian, K.; Wu, X.; Giacca, A.; Sun, Y. A monolithic polymeric microdevice for $\mathrm{pH}$-responsive drug delivery. Biomed. Microdevices 2009, 11, 1251-1257.

61. Qiu, Y.; Park, K. Environment-sensitive hydrogels for drug delivery. Adv. Drug Delivery Rev. 2001, 53, 321-339.

62. Park, H.; Park, K. Biocompatibility issues of implantable drug delivery systems. Pharm. Res. 1996, 13, 1770-1776.

63. Hsu, S.-H.; Tseng, H.-J. In vitro biocompatibility of PTMO-based polyurethanes and those containing PDMS blocks. J. Biomat. Appl. 2004, 19, 135-146. 
64. Kurian, P.; Kasibhatla, B.; Daum, J.; Burns, C.A.; Moosa, M.; Rosenthal, K.S.; Kennedy, J.P. Synthesis, permeability and biocompatibility of tricomponent membranes containing polyethylene glycol, polydimethylsiloxane and polypentamethylcyclopentasiloxane domains. Biomaterials 2003, 24, 3493-3503.

65. Sanders, R.S.; Lee, M.T. Implantable pacemakers. Proc. IEEE 1996, 84, 480-486.

66. Kane, M.J.; Breen, P.P.; Quondamatteo, F.; ÓLaighin, G. BION microstimulators: A case study in the engineering of an electronic implantable medical device. Med. Eng. Phys. 2011, 33, 7-16.

67. Ferrara, L.; Fleischman, A.; Dunning, J.; Zorman, C.; Roy, S. Effects of biomedical sterilization processes on performance characteristics of MEMS pressure sensors. Biomed. Microdevices 2007, 9, 809-814.

68. Voskerician, G.; Shive, M.S.; Shawgo, R.S.; Recum, H.V.; Anderson, J.M.; Cima, M.J.; Langer, R. Biocompatibility and biofouling of MEMS drug delivery devices. Biomaterials 2003, 24, 1959-1967.

69. Wisniewski, N.; Moussy, F.; Reichert, W.M. Characterization of implantable biosensor membrane biofouling. Fresenius J. Anal. Chem. 2000, 366, 611-621.

70. Reuben, B.G.; Perl, O.; Morgan, N.L.; Stratford, P.; Dudley, L.Y.; Hawes, C. Phospholipid coatings for the prevention of membrane fouling. J. Chem. Technol. Biotechnol. 1995, 63, 85-91.

71. Clausen, I.; Seeberg, T.M.; Gheorghe, C.; Wang, D.T. Biofouling on Protective Coatings for Implantable MEMS. In IEEE Sensors, Kona, HI, USA, 1-4 November 2010; pp. 751-754.

72. Schmehl, J.M.; Harder, C.; Wendel, H.P.; Claussen, C.D.; Tepe, G. Silicon carbide coating of nitinol stents to increase antithrombogenic properties and reduce nickel release. Cardiovasc. Revascularization Medicine 2008, 9, 255-262.

73. Singh, R.A.; Siyuan, L.; Satyanarayana, N.; Kustandi, T.S.; Sinha, S.K. Bio-inspired polymeric patterns with enhanced wear durability for microsystem applications. Mat. Sci. Eng. C 2011, 31, 1577-1583.

74. Subhash, G.; Corwin, A.; de Boer, M. Evolution of wear characteristics and frictional behavior in MEMS devices. Tribology. Lett. 2011, 41, 177-189.

75. Hongbin, Y.; Guangya, Z.; Sinha, S.K.; Leong, J.Y.; Fook Siong, C. Characterization and reduction of MEMS sidewall friction using novel microtribometer and localized lubrication method. J. Microelectromechanical. Syst. 2011, 20, 991-1000.

76. Wang, Y.; Wang, L.; Xue, Q.; Yuan, N.; Ding, J. A facile method to improve tribological properties of silicon surface by combining nanogrooves patterning and thin film lubrication. Colloid. Surface. A 2010, 372, 139-145.

77. Mercanzini, A.; Cheung, K.; Buhl, D.L.; Boers, M.; Maillard, A.; Colin, P.; Bensadoun, J.-C.; Bertsch, A.; Renaud, P. Demonstration of cortical recording using novel flexible polymer neural probes. Sens. Actuator. A: 2008, 143, 90-96.

78. Polikov, V.S.; Tresco, P.A.; Reichert, W.M. Response of brain tissue to chronically implanted neural electrodes. J. Neurosci. Meth. 2005, 148, 1-18.

79. Lee, K.; Singh, A.; He, J.; Massia, S.; Kim, B.; Raupp, G. Polyimide based neural implants with stiffness improvement. Sens. Actuator. B 2004, 102, 67-72.

80. Gumbiner, B.M. Cell adhesion: The molecular basis of tissue architecture and morphogenesis.

Cell 1996, 84, 345-357. 
81. Yuli, W.; Jeng-Hao, P.; Hsuan-Hong, L.; Christopher, E.S.; Mark, B.; Li, G.P.; Nancy, L.A. Surface graft polymerization of SU-8 for bio-MEMS applications. J. Micromechanic. Microengineer. 2007, 17, 1371-1380.

82. Bouaidat, S.; Winther-Jensen, B.; Christensen, S.F.; Jonsmann, J. Plasma-polymerized coatings for bio-MEMS applications. Sens. Actuator. A 2004, 110, 390-394.

(C) 2012 by the authors; licensee MDPI, Basel, Switzerland. This article is an open access article distributed under the terms and conditions of the Creative Commons Attribution license (http://creativecommons.org/licenses/by/3.0/). 